\title{
'||||||||||||||||||||||||||||||||||||||||||||||||||||||||||||||||||.
}

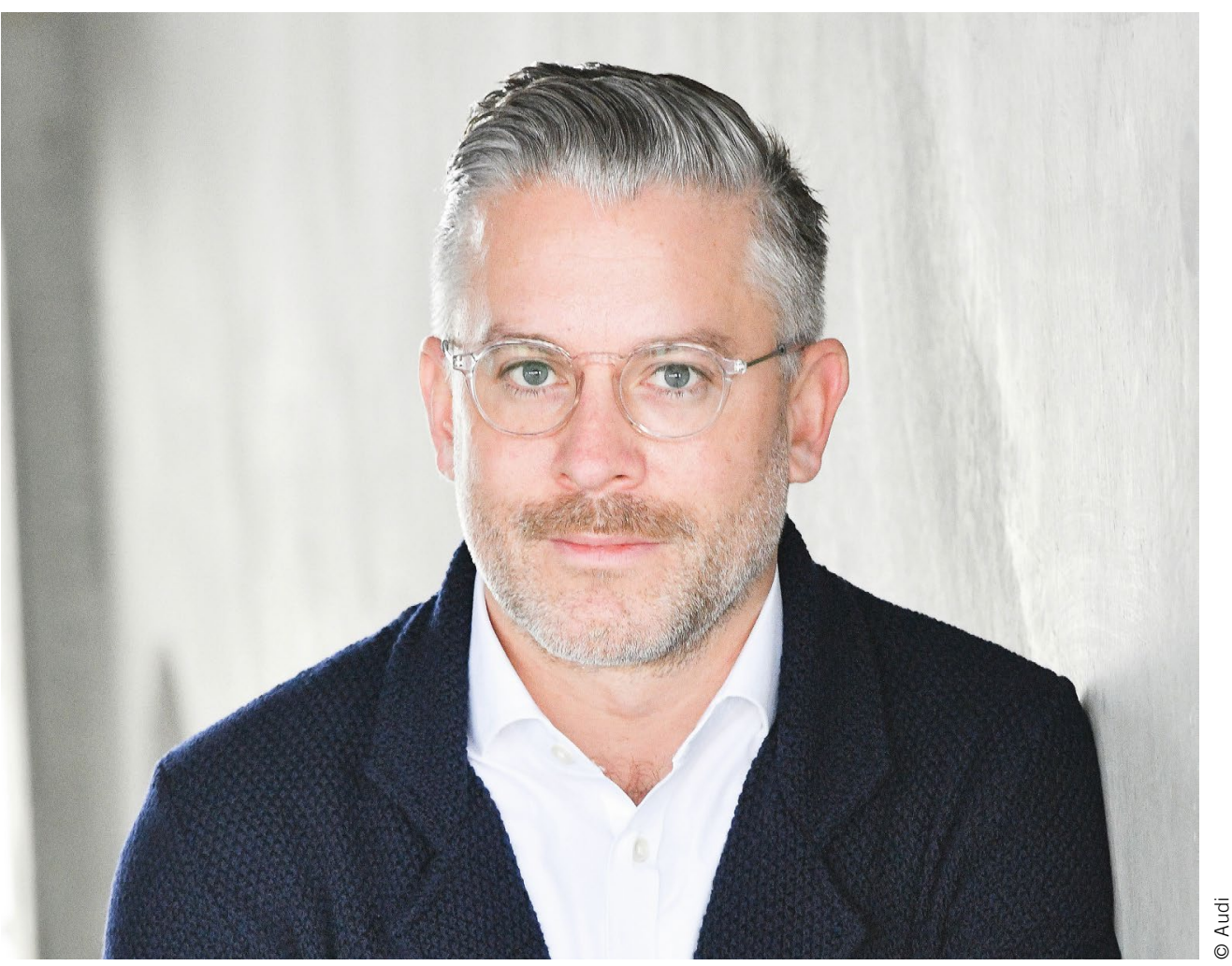

Dipl.-Bw. Marco Philippi, MBA

Senior Director

Procurement Strategy

of Audi AG

\section{The Opportunity of Chemical Recycling}

Audi is present in more than 100 markets and produces automobiles at 13 locations. Our supplier network comprises 14,000 direct suppliers from more than 60 countries. Changes that we initiate have a major impact on all those who are part of our network. We can achieve a great deal together. With its suppliers, Audi is placing an emphasis on the efficient and economical use of the resources we need for manufacturing our products. Our ambition is to be a pioneer with regard to handling these recyclable materials. Our goal is to establish closed-loop systems and obtain new recyclable materials from waste. I firmly believe that our industry must put sustainability at the core of its philosophy in order to be successful in the future.

The pilot project for chemical recycling is a prime example of this goal. It is designed to demonstrate that technology and innovation can lead to greater sustainability in Germany as a business location. In the "Industrial Resource Strategies" think tank at the Karlsruhe Institute of Technology (KIT), university science and entrepreneurship are working together with assistance from policymakers. The objectives of chemical recycling are ambitious: We want to transfer mixed plastic fractions from the automotive sector back into a resource-conserving loop and, in contrast to mechanical reprocessing, we want to do so at the high quality level of new goods. The procedure opens up additional potential for the resource-conserving purchase and handling of recyclable materials in the automobile. Initial pyrolysis tests are to determine the conditions under which chemical recycling of used plastics is possible. The focus of these tests will be on the effect of paint, contamination, or unwanted plastics in particular. At the same time, we will analyze the efficiency and possible environmental effects in the event of scaling.

If we succeed in demonstrating technical feasibility while taking into account ecological and economic premises, we will establish the process for suitable plastic fractions in cooperation with partners from the chemical industry. This gives us the opportunity to keep as large a percentage of our own plastics in the loop as possible and thereby save primary resources in the future. Audi has already had good experiences with these kinds of developments. For example, we closed the loop for aluminum together with our suppliers. This enabled us to avoid around 150,000 metric tons of $\mathrm{CO}_{2}$ on balance and save valuable resources in 2019 alone.

We are convinced that similar success can also be achieved with plastics. These are crucial steps on the path toward the future viability of sustainable mobility - and therefore a common interest of business, politics, and society. 